This item was submitted to Loughborough's Research Repository by the author.

Items in Figshare are protected by copyright, with all rights reserved, unless otherwise indicated.

\title{
Differences in plantarflexor function during a stretch-shortening cycle task due to limb preference
}

\section{PLEASE CITE THE PUBLISHED VERSION}

http://dx.doi.org/10.1080/1357650X.2014.921688

\section{PUBLISHER}

(c) Taylor \& Francis

\section{VERSION}

AM (Accepted Manuscript)

\section{PUBLISHER STATEMENT}

This work is made available according to the conditions of the Creative Commons Attribution-NonCommercialNoDerivatives 4.0 International (CC BY-NC-ND 4.0) licence. Full details of this licence are available at: https://creativecommons.org/licenses/by-nc-nd/4.0/

\section{LICENCE}

CC BY-NC-ND 4.0

\section{REPOSITORY RECORD}

Furlong, Laura-Anne, and Andrew J. Harrison. 2019. "Differences in Plantarflexor Function During a Stretchshortening Cycle Task Due to Limb Preference". figshare. https://hdl.handle.net/2134/25222. 
Differences in plantarflexor function during a stretch-shortening cycle task due to limb preference

$$
\text { Laura-Anne M Furlong and Andrew J Harrison }
$$

Biomechanics Research Unit, Department of Physical Education and Sport Sciences, University of Limerick, Ireland

\section{Corresponding author: \\ Laura-Anne M Furlong \\ laura-anne.furlong@ul.ie}

Shortened title: Plantarflexor function and limb preference 


\section{Abstract}

Most healthy humans move symmetrically at gross limb level but large kinetic and kinematic asymmetries have been observed at joint level during locomotion. The aim of this study was to assess muscle function asymmetries in healthy, active adults using an adapted force sledge apparatus which isolates the plantarflexors during a stretch-shortening cycle task. Peak force, rate of force development and stretch-shortening cycle function of preferred and non-preferred limbs were assessed in 21 healthy, active individuals using the adapted sledge and three-dimensional motion analysis. Between-limb differences and relationships were determined using paired t-tests/Wilcoxon-Signed rank test, Cohen's $d_{z}$, absolute symmetry index and Pearson's r/Spearman's rho. Significant differences with moderate effect size (ES) were observed in peak force (ES: 0.66), rate of peak force development (ES: 0.78), rate of force development in the first 50 ms (ES: 0.76), flight time (ES: 0.64) and stretch-shortening cycle function (0.68), with no difference in contact time or duration of eccentric loading. A small ES (0.56) was observed in rate of force development in the first $30 \mathrm{~ms}$. The upper range of asymmetry observed (up to 44.9\%) was larger than previously reported for healthy individuals, indicating compensations occur at proximal joints during locomotion to ensure symmetrical movement.

\section{Keywords}

Asymmetry, limb dominance, dynamical systems, running, injury

\section{Acknowledgements}

The authors would like to thank the Irish Research Council for funding this study under the Embark Initiative. 


\section{Introduction}

The assumption of limb symmetry in movement is reasonable as most healthy individuals walk and run without a limp or visible evidence of asymmetry (Flanagan \& Harrison, 2007). However, there is a growing body of evidence to suggest the legs behave differently in several kinematic and kinetic measures during dynamic tasks. An understanding of these asymmetries and establishment of the level of asymmetry in healthy individuals is important to avoid potential injury (Knapik, Bauman, Jones, Harris, \& Vaughan, 1991) and aid in the monitoring of rehabilitation programmes. Knowledge of the kinematic and kinetic characteristics of the weaker or lesser skilled limb is also important in guiding skill development and enhancing performance (Ball, 2011). Kinematic and kinetic asymmetries in healthy individuals have been observed at the ankle, knee and hip during walking and running tasks by several authors (Bredeweg, Buist, \& Kluitenberg, 2013; Exell, Irwin, Gittoes, \& Kerwin, 2012; Korhonen et al., 2010). How the body coordinates these asymmetric and variable structures to produce movement is known as the degrees of freedom problem of movement coordination and control (van Emmerik, Rosenstein, McDermott, \& Hamill, 2004). The traditional view is that variability and asymmetry represent biological noise but Davids, Glazier, Araújo, and Bartlett (2003) suggested these measures may be better viewed as the body's response to the constraints imposed during performance and instead reflect large adaptive capacities and flexibility (Holt, Jeng, Ratcliffe, \& Hamill, 1995).

Asymmetry in lower limb function may be due to a variety of factors including lateralisation (Gabbard \& Hart, 1996), sport and training background (Cavanagh, Pollock, \& Landa, 1977; Cromie, Greenwood, \& McCullagh, 2007), injury (Schiltz et al., 2009; Zifchock, Davis, \& Hamill, 2006), speed of movement (Cavagna, 2006; Schiltz et al., 2009), age (Atkins, Hesketh, \& Sinclair, 2013), muscle activation (Valderrano et al., 2007) or contextual interference (Vagenas \& Hoshizaki, 1992). Quantification of limb function asymmetry and its underlying mechanisms is difficult for several reasons. It is a highly individualised and variable measure as people respond differently to greater demands on the musculoskeletal system (Smak, Neptune, \& Hull, 1999) and any asymmetry observed is highly dependent on the test used (Jones \& Bampouras, 2010). As different tests of limb and muscle 
function give different values for asymmetry, assessments of between-limb differences must be valid for the task of interest. Tests must demonstrate similar contraction modes and kinematics as the task of interest to avoid erroneous detection of asymmetry which is not present during performance of dynamic tasks (Menzel et al., 2013).

The plantarflexors are a vital muscle group in locomotion, acting to both produce force and stabilise the shank upon landing (Kuitunen, Komi, \& Kyrolainen, 2002). They act on the ankle joint, which is one of the most commonly injured areas of the lower limb in sport (Fong, Hong, Chan, Yung, \& Chan, 2007; Kolt, 2013). Running and sprinting consist of repeated stretch-shortening cycles (SSC) i.e. cyclical eccentric contraction prior to concentric contraction. Previous work using a force sledge found no significant between-limb differences in peak force generation and SSC function of the entire lower limb in a cyclical loading task (Flanagan \& Harrison, 2007), but this may be due to compensatory motor control strategies rather than symmetrical behaviour at individual joint or muscle level. An upper limit of $15 \%$ asymmetry in healthy limbs has been previously suggested (Knapik et al., 1991), but there is no research on isolated plantarflexor function in a SSC task to inform clinical decision-making on this.

Muscle function asymmetry assessment requires high levels of test validity, utilising similar contraction modes and range of motion as the task of interest. Furlong and Harrison (2013) developed a reliable, valid method of measuring plantarflexor muscle-tendon unit (MTU) behaviour during a dynamic, fast SSC activity (i.e. contact time of less than 0.25 s). The task uses similar ankle kinematics and contact times as observed during running studies, with similar rates of peak force development reported as those observed in the Achilles tendon during walking (Finni, Komi, \& Lukkariniemi, 1998). The aim of this study was to identify between-limb differences and level of asymmetry in plantarflexor muscle function of healthy, active adults during a dynamic SSC task using this adapted force sledge. This may help to explain observed asymmetries in kinematic and kinetic parameters during gait, as well as indicate if compensation occurs in the lower limb during a cyclical task to ensure symmetrical movement. 


\section{Method}

\section{Participants}

Following institutional ethical approval, 21 active individuals gave written, informed consent to participate in this study (10 females, 11 males; age: $23.8 \pm 2.27$ years; mass: $72.5 \pm 11.9 \mathrm{~kg}$; height: $1.72 \pm .083 \mathrm{~m}$ ). None had a history of lower limb surgery and all were injury free in the lower limb for the preceding 3 months. Participants were advised to refrain from unaccustomed strenuous activity for the 24 hours preceding data collection.

\section{Test protocol}

An adapted force sledge was used to obtain measures of plantarflexor force, force development and stretch-shortening cycle function. A six camera 3D motion analysis system (500 Hz, MAC Eagle, Motion Analysis Corporation Inc., Santa Rosa, CA., USA) was used to track $9.5 \mathrm{~mm}$ retro-reflective markers on the sledge plate edge, the lateral $5^{\text {th }}$ metatarsophalangeal joint (5MTP), malleolus (MALL) and estimated knee joint centre (KJC). Participants were positioned supine at the base of the sledge as shown in figure 1 . They were instructed to strike the sledge foot plate in a marked area as rhythmically as possible, pushing it as high as they could while minimising contact time using only their ankle joint. Throughout this, the hip angle was approximately $135^{\circ}$ and knee angle between $140^{\circ}$ and $160^{\circ}$. Familiarisation consisted of approximately 25-30 impacts with no added mass where the subject initially pushed the plate away from them and struck it rhythmically and a second trial where the plate was released from $30 \mathrm{~cm}$ away from the foot. The protocol continued until the subject was satisfied that they were familiar with the task and the researcher deemed the subject was consistently striking the sledge plate as instructed. Previous work has shown that subsequent trials at a variety of loadings resulted in low coefficient of variation and high intra-class correlation coefficients during each trial for the variables of interest (Furlong \& Harrison, 2013). This shows participants were sufficiently familiar with the task to produce reliable data even after 25-30 impacts.

$<$ Insert Fig. 1 about here $>$ 
The two limbs were tested sequentially, with the preferred leg familiarised and tested first as participants were more comfortable completing testing in this order. Pilot work showed the order of limb testing did not introduce bias into the data. Due to the nature of the task, the preferred leg was defined as the preferred leg for single-leg hopping.. For all participants, this was also the preferred leg for kicking a ball. The sledge plate was released from $30 \mathrm{~cm}$ above the foot following a ' $3,2,1$ ' countdown. The same instructions were given as in familiarisation. The plate was secured away from the foot after successful completion of each trial and additional mass added to the sledge. Participants took a minimum of two minutes break between trials to minimise fatigue effects. If participants felt they needed longer to recover, this was allowed. The test was administered similar to an 11 repetition maximum (11RM) strength test, with the researcher attempting to reach the maximum loading in as short a time frame as possible. At least 90 impacts were completed per leg prior to data collection. Test load was approximately 70\% of this 11RM (actual test load:17.4 $\pm 3.9 \mathrm{~kg}, 24.4 \pm 6.2 \%$ body mass, $71.2 \pm 5.28 \% 11 \mathrm{RM})$ as this has been shown to be the most reliable for the variables of interest (Furlong \& Harrison, 2013). The same protocol was used for the non-preferred leg with the same mass used for the test trial.

\section{Data processing and analysis}

Residual analyses were conducted to identify the optimum marker cut-off frequency and ensure an optimal signal: noise ratio (Winter, 2005). Markers were filtered using a fourth order, zero lag, low-pass Butterworth filter with cut-off frequencies of $12 \mathrm{~Hz}$ for the sledge and 5MTP markers, and $14 \mathrm{~Hz}$ and $18 \mathrm{~Hz}$ for the MALL and KJC markers respectively. A visual check was used to ensure these cut-off frequencies did not over-smooth the impact event. Ankle angle was defined as the sagittal plane angle formed by the 5MTP, MALL and KJC markers, with the duration of eccentric loading defined as the percentage of contact time where the foot was in dorsiflexion. Angles were exported directly from the motion analysis software (Cortex v3.1.1.1290, Motion Analysis Corporation Inc., Santa Rosa, CA., USA) with all other variables calculated in Microsoft Excel (Microsoft Inc., Redmond, WA., USA). 
Plate acceleration was calculated as the second derivative of plate position with respect to time, with force calculated using Newton's second law. A correction for the component of weight acting down the sledge rails was used since the sledge was inclined at $30^{\circ}$. The difference between calculated average acceleration and predicted acceleration at $30^{\circ}$ inclination during free-fall $\left(4.903325 \mathrm{~m} . \mathrm{s}^{-2}\right)$ has previously been calculated as $0.00928 \mathrm{~m} . \mathrm{s}^{-2}(0.189 \%)$ therefore frictional force was considered to be negligible in calculations. Peak force $\left(\mathrm{F}_{\mathrm{P}}\right)$ was the maximum force developed during each contact time with rate of peak force development (RPFD) calculated as the $F_{P}$ divided by the time in seconds to reach $F_{p}$. Rate of force development in the first 30 and $50 \mathrm{~ms}\left(\mathrm{RFD}_{0-30}, \mathrm{RFD}_{0-50}\right)$ was calculated as the force value at $30 \mathrm{~ms}$ and $50 \mathrm{~ms}$ post-contact, divided by .03 or .05 s respectively. Contact time (CT) was defined as the period when plate marker acceleration was greater than zero and flight time (FT) defined as the period between consecutive CT. Plate height (i.e. displacement from release to peak of flight) was calculated using the equations of motion and assumed equal periods of upwards and downwards flight. The reactive strength index (RSI) refers to the ability of a subject to utilise the SSC, with increased RSI considered indicative of an increased ability to transition quickly from an eccentric to concentric contraction (Young, 1995). It was defined as the ratio between plate height and preceding CT.

The middle impacts (\#5 to \#7) were selected for analysis. Absolute symmetry index (ASI) was calculated as

$$
A S I=\frac{\left|\left(X_{P}-X_{N P}\right)\right|}{\frac{1}{2}\left(X_{P}+X_{N P}\right)} \times 100 \%
$$

Karamanidis, Arampatzis, and Bruggemann (2003)

Where $\mathrm{X}$ is the measure of interest and $\mathrm{P}$ and NP refer to the preferred and non-preferred legs respectively. The strength of this method is that it quantifies absolute difference between limbs rather than allowing positive and negative differences cancel each other out. A score of $0 \%$ indicates perfect symmetry between limbs.

\section{Limb anthropometrics}


As the effect of muscle size on force output is established, calf circumference to the closest millimetre was obtained at a number of points using a flexible tape measure (Rollfix, Hoechstmass, Germany). Circumference was considered a simple, easily obtained measure which was correlated to plantarflexor muscle size (Bamman, Newcomer, Larson-Mayer, Weinsier, \& Hunter, 2000). The same experienced researcher obtained all circumferences. Participants stood with the feet hip width apart and malleoli level on a $50 \mathrm{~cm}$ box so the researcher could obtain measures at eye level to avoid parallax error. Measures were obtained in the plane perpendicular to the long axis of the shank. Shank length was defined as the distance between the estimated knee joint centre and the lateral malleolus. Limb circumferences were recorded at a point immediately inferior to the patella, at positions $25 \%$, $50 \%$ and $75 \%$ from the proximal end of the segment, at the widest point of the calf and immediately superior to the malleolus. Five measures were recorded for each variable from each leg with the average used for later analysis.

\section{Statistical analysis}

Statistical analysis was completed in SPSS Statistics 20 (IBM, Armonk, NY, USA). The assumptions of normality and homogeneity of variance were assessed using Shapiro-Wilk’s test and Levene’s test. When a variable did not satisfy these assumptions, Wilcoxon's signed rank test was used to determine if differences existed between the preferred and non-preferred leg. For all other variables, paired samples t-tests were used. Pearson's $r$ and Spearman's rho were calculated to determine the strength of the linear relationship between the measures obtained from the two limbs. Correlation coefficients were considered large when the correlation coefficient was.5 to.7 and very large when between.7 and.9 (Hopkins, 2006). The level of significance for all tests was set at $\alpha<0.05$. Effect size was calculated using Cohen's $d_{z}$ using the formula

$$
\mathrm{d}_{\mathrm{z}}=\frac{\mathrm{m}_{\mathrm{z}}}{\sigma_{\mathrm{z}}}
$$

where $\mathrm{m}_{\mathrm{z}}=$ the mean of the observed between-limb differences and $\sigma_{\mathrm{z}}=$ the standard deviation of the observed differences (Cohen, 1977). The scale for classification of effect size was 0.2 to $0.6=$ small, 
0.6 to 1.2 = medium, 1.2 and above = large (Hopkins, 2006). Where between-limb anthropometrics were significantly different, $\mathrm{F}_{\mathrm{P}}, \mathrm{RPFD}, \mathrm{RFD}_{0-30}$ and $\mathrm{RFD}_{0-50}$ were calculated normalised to the relevant circumference and between-limb differences and relationship recalculated.

\section{$\underline{\text { Results }}$}

Ankle angle data was not obtained from three participants due to problems tracking the malleolus marker with the position of the sledge but for the remaining 18 participants, no significant difference was observed between legs in the duration of eccentric loading on the muscle. No differences were observed in CT or most of the anthropometric measures. Limb circumference at the widest point of the calf was significantly different $(p=0.022$, preferred leg: $0.363 \pm 0.018 \mathrm{~m}$, non-preferred leg: 0.361 \pm 0.019 m, ASI: $1.1 \pm 0.73 \%$, moderate effect size). Statistically significant differences in $\mathrm{F}_{\mathrm{P}}$, RPFD, $\mathrm{RFD}_{0-30}, \mathrm{RFD}_{0-50}$, FT and RSI were observed with large ASI from $11.4 \pm 10.87$ to $25.3 \pm 19.6 \%$. Effect sizes were small for $\mathrm{RFD}_{0-30}$ and moderate for the remaining measures. Significant differences with similar effect sizes and ASI were still present after normalisations to maximum calf width therefore only absolute values are presented in Table 1 . Variability observed was almost the same magnitude as the average observed value. All correlation coefficients were statistically significant, with large to very large linear relationships observed between the preferred and non-preferred limbs.

\section{Discussion}

The results lend strong support to the presence of asymmetry in measures of plantarflexor MTU function during a dynamic, controlled, SSC task. Asymmetries were observed in all measures of force, force development and SSC function without asymmetry in contact time or duration of eccentric loading, and most measures of estimated muscle size. Differences observed were hence due to differences in physiological and mechanical behaviour rather than altered kinematic strategies or muscle mass. The magnitude of difference in circumference of the two limbs was very small so it is not surprising that normalised data resulted in the same conclusion. Asymmetry in plantarflexor function has previously been observed (Valderrano et al., 2007) but the levels of asymmetry observed here (from zero to almost $45 \%$ ) are greater than the $15 \%$ previously reported as the upper limit of 
asymmetry for healthy limb function (Knapik et al., 1991). This has implications for our understanding of natural variation in function between limbs, and recommendations for rehabilitation. It appears there is a greater range of acceptable asymmetry in measures of MTU function than previously thought as none of these participants proceeded to develop any plantarflexor or Achilles tendon problems in the 12 months following data collection. These results support the conclusions of Davids et al. (2003) who stated that even though measures of function may be considered abnormal when compared to the ideal medical model, they may still be healthy but reflect the adaptations of the limb to the constraints imposed during performance of the task. This large range also highlights the individualised nature of asymmetry.

The large correlations observed are of interest as the correlation coefficients for all measures of force, force development and RSI were greater than .59, i.e. for every 1 standard deviation change in the preferred limb there will be at least a .59 standard deviation change in the non-preferred limb (Hopkins, 2006). This suggests that despite large differences the MTU still behaved similarly, i.e. force production in both limbs may be high, even if there is a difference between them. In healthy adults, this would be expected. Lower correlation may indicate injury or previous injury as the relationship between the healthy and injured limb may vary considerably. While the healthy limb may perform well on a task, the corresponding score from an injured limb may vary significantly dependent on extent of damage and rehabilitation.

The strength of this study is that it is one of the first to test plantarflexor muscle function asymmetries in a valid, dynamic test condition which other authors have highlighted as a major limitation of methods of assessing limb asymmetry (Menzel et al., 2013). Despite the high level of control afforded by dynamometry, it lacks similar loading patterns to SSC tasks in magnitude of forces, kinematics and impact. Accounting for a typical lever length of $16-18 \mathrm{~cm}$ from the malleolus to the ball of the foot, preferred force values observed here are greater than the concentric plantarflexor torque of 41.8 N.m.

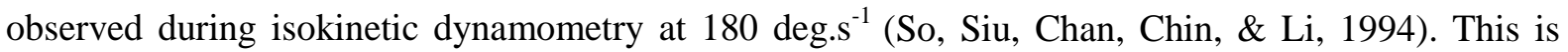
most likely due to the increased involvement of the SSC during the task and increased time for force development. The results of this study may help explain the asymmetries observed by other authors 
during fast SSC activities. Exell et al. (2012) reported ankle joint work asymmetries of up to 93\%. Joint work, as the time integral of joint power, is dependent on the product of joint torque and joint angular velocity which is subsequently dependent on speed of muscular contraction, rate of force development and opposing co-contraction. Altered joint torque may be the result of altered muscle group force production capabilities, and angular velocity can be determined by speed of muscle contraction, rate of force development and opposing co-contraction. However, previous work has shown altered proprioceptive capacities of the lower limb depending on whether alternating or bipedal hopping strategies are used (Travers, Debenham, Gibson, Campbell, \& Allison, 2013). This may affect muscle pre-activation strategies prior to impact and potentially, observed force and force development values. Therefore, further study is required to identify how well the observed asymmetries in muscle function correspond to kinematic and kinetic asymmetries during activities such as running. The human body can utilise a number of different degrees of freedom to produce the same output, and similarly utilise the same degrees of freedom coordinated differently to produce different output (Heiderscheit, 2000). The results of this study clearly show a difference in output, assessed by plate flight time. These differences are most likely related to the differences observed in the force and force development capabilities of the plantarflexor muscle group as no differences were observed in the plate contact time or duration of eccentric loading on the muscle which are indicative of the strategy used by the participant to project the plate. Flanagan and Harrison (2007) found no significant between-leg differences in measures of peak force generated or RSI of the entire lower limb during repetitive cyclical loading. Similarly, Buckeridge, Hislop, Bull, and McGregor (2012) reported no significant differences in lumbar-pelvic kinematics during rowing, despite observed between-leg differences in hip and knee kinematics, suggesting similar movements at the proximal limb do not reflect distal kinematics and kinetics at individual joint level. The results of this study show the plantarflexors of preferred and non-preferred limbs behave differently in their forceproduction capabilities, how quickly they develop force and the outcome of force application. This suggests the hip, knee and ankle joints of the lower limb compensate for each other to maintain overall lower limb symmetry during cyclical activities such as running. Previous work has shown differences in individual joint contributions to overall limb performance dependent on task demands 
(Hobara, Inoue, Omuro, Muraoka, \& Kanosue, 2011), so it is probable that the mechanisms of compensation also vary with task.

The idea of lower-limb compensation is in agreement with Exell et al. (2012) who reported large asymmetries in hip, knee and ankle kinematics and kinetics during maximal velocity sprinting. This supports previous findings suggesting the legs use different strategies to get off the ground (Sadeghi, Allard, Prince, \& Labelle, 2000). Ball (2011) reported higher angular velocities at the distal joints of the preferred limb during kicking and lower angular velocities at the proximal joints compared to the non-preferred limb, which is consistent with Bernstein’s (1967) theory of motor control. This shows the ability of the two limbs to adapt differently to the conditions imposed and supports the idea of compensation in each limb to ensure successful performance. The regular preferential selection of one limb for performance of a dynamic task results in alterations in limb muscle function. During hopping or kicking, the preferred leg is used for a quicker movement with greater motor control and force development requirements while the non-preferred leg utilises more isometric muscular contractions. This provides further evidence for the flexibility of the body to adapt to the constraints imposed to maintain symmetrical movement.

\section{Conclusions}

This study has shown significant differences in plantarflexor MTU function during a dynamic, fast SSC task. The range of asymmetry is much greater than the $15 \%$ upper limit recommended by athletic trainers and healthcare professionals as the upper limit in healthy individuals. The wide range of asymmetry observed highlights the highly individualised nature of this particular measure, and indicates compensation may occur at the proximal joints to ensure symmetrical movement at gross limb level. These results may also help to explain the high levels of kinematic and kinetic asymmetry observed during running and sprinting. 


\section{$\underline{\text { References }}$}

Atkins, SJ, Hesketh, C, \& Sinclair, JK. (2013). The presence of bilateral imbalance of the lower limbs in elite youth soccer players of different ages. Journal of Strength and Conditioning Research. doi: 10.1519/JSC.0b013e3182987044

Ball, KA. (2011). Kinematic comparison of the preferred and non-preferred foot punt kick. Journal of Sports Sciences, 29(14), 1545-1552.

Bamman, MM, Newcomer, BR, Larson-Mayer, D Ennette, Weinsier, RL, \& Hunter, GR. (2000). Evaluation of the strength-size relationship in vivo using various muscle size indices. Medicine and Science in Sports and Exercise, 32(7), 1307-1313.

Bernstein, N. (1967). The coordination and regulation of movement London: Pergamon Press.

Bredeweg, S. W., Buist, I., \& Kluitenberg, B. (2013). Differences in kinetic asymmetry between injured and noninjured novice runners: A prospective cohort study. Gait \& Posture, 38(4), 847-852.

Buckeridge, E, Hislop, S, Bull, A. M. J., \& McGregor, A (2012). Kinematic asymmetries of the lower limbs during ergometer rowing. Medicine and Science in Sports and Exercise, 44(11), 2147-2153.

Cavagna, GA. (2006). The landing-take-off asymmetry in human running. Journal of Experimental Biology, 209, 4051-4060.

Cavanagh, PR, Pollock, ML, \& Landa, J. (1977). A biomechanical comparison of elite and good distance runners. Annals of the New York Academy of Sciences, 301, 328-345.

Cohen, J. (1977). Statistical Power Analysis for the Behavioral Sciences (Revised ed.). New York: Academic Press Inc.

Cromie, S, Greenwood, JG, \& McCullagh, JF. (2007). Does Irish-dance training influence lower-limb asymmetry? Laterality, 12(6), 500-506.

Davids, K, Glazier, P, Araújo, D, \& Bartlett, R. (2003). Movement systems as dynamical systems: the functional role of variability and its implications for sports medicine. Sports Medicine, 33(4), 245-260.

Exell, TA, Irwin, G, Gittoes, MJR, \& Kerwin, DG. (2012). Implications of intra-limb variability on asymmetry analyses. Journal of Sports Sciences, 30(4), 403-409.

Finni, T, Komi, PV, \& Lukkariniemi, J. (1998). Achilles tendon loading during walking: application of a novel optic fiber technique. European Journal of Applied Physiology, 77, 289-291.

Flanagan, EP, \& Harrison, AJ. (2007). Muscle dynamics differences between legs in healthy adults. Journal of Strength and Conditioning Research 21(1), 67-72.

Fong, DTP, Hong, Y, Chan, LK, Yung, PSH, \& Chan, KM. (2007). A systematic review on ankle injury and ankle sprains in sports. Sports Medicine, 37(1), 73-94.

Furlong, LAM, \& Harrison, AJ. (2013). Reliability and consistency of plantarflexor stretch-shortening cycle function using an adapted force sledge apparatus. Physiological Measurement, 34(4), 437-448.

Gabbard, C, \& Hart, S. (1996). A question of foot dominance. The Journal of General Psychology, 123(4), 289-296.

Heiderscheit, B (2000). Movement variability as a clinical measure for locomotion. Journal of Applied Biomechanics, 16, 419-427. 
Hobara, H, Inoue, K, Omuro, K, Muraoka, T, \& Kanosue, K. (2011). Determinant of leg stiffness during hopping is frequency-dependent. European Journal of Applied Physiology, 111, 21952201.

Holt, KG, Jeng, SF, Ratcliffe, R, \& Hamill, J. (1995). Energetic cost and stability during human walking at the preferred stride frequency. Journal of Motor Behavior, 27(2), 164-178.

Hopkins, WG. (2006). A New View of Statistics: A Scale of Magnitudes for Effect Sizes. Retrieved January 9, 2014

Jones, PA, \& Bampouras, TM. (2010). A comparison of isokinetic and functional methods of assessing bilateral strength imbalance. Journal of Strength and Conditioning Research, 24(6), 1553-1558.

Karamanidis, K., Arampatzis, A., \& Bruggemann, G. P. (2003). Symmetry and reproducibility of kinematic parameters during various running techniques. Medicine and Science in Sports and Exercise, 35(6), 1009-1016.

Knapik, J, Bauman, C, Jones, B, Harris, J, \& Vaughan, L. (1991). Preseason strength and flexibility imbalances associated with athletic injuries in female collegiate athletes. American Journal of Sports Medicine, 19, 76-81.

Kolt, G. (2013). Research on the ankle in sport. Journal of Science and Medicine in Sport, 16(5), 387.

Korhonen, MT, Suominen, H., Viitasalo, JT, Liikavainio, T, Alen, M, \& Mero, A. (2010). Variability and symmetry of force platform variables in maximum-speed running in young and older athletes. Journal of Applied Biomechanics, 28(357-368).

Kuitunen, S , Komi, PV , \& Kyrolainen, H (2002). Knee and ankle joint stiffness in sprint running. Medicine and Science in Sports and Exercise, 34(1), 166-173.

Menzel, H.-J., Chagas, MH, Szmuchrowski, LA, Araujo, SRS, Andrade, AGP de, \& de JesusMoraleida, FR. (2013). Analysis of lower limb asymmetries by isokinetic and vertical jump tests in soccer players. Journal of Strength and Conditioning Research, 27(5), 1370-1377.

Sadeghi, H, Allard, P, Prince, F, \& Labelle, H. (2000). Symmetry and limb dominance in able-bodied gait: a review. Gait and Posture, 12, 34-45.

Schiltz, M, Lehance, C, Maquet, D, Bury, T, Crielaard, J.-M., \& Croisier, J.-L. (2009). Explosive strength imbalances in professional basketball players. Journal of Athletic Training, 44(1), 39-47.

Smak, W, Neptune, RR, \& Hull, ML. (1999). The influence of pedaling rate on bilateral asymmetry in cycling. Journal of Biomechanics, 32, 899-906.

So, C.-H., Siu, TO, Chan, KM, Chin, MK, \& Li, CT. (1994). Isokinetic profile of dorsiflexors and plantarflexors of the ankle - a comparative study of elite versus untrained subjects. British Journal of Sports Medicine, 28(1), 25-30.

Travers, MJ, Debenham, J, Gibson, W, Campbell, A, \& Allison, GT. (2013). Stability of lower limb minimal perceptible difference in floor height during hopping stretch-shortening cycles. Physiological Measurement, 34, 1375-1386. doi: 10.1088/0967-3334/34/10/1375

Vagenas, G., \& Hoshizaki, B. (1992). A multivariable analysis of lower-extremity kinematic asymmetry in running. International Journal of Sport Biomechanics, 8(1), 11-29.

Valderrano, V, Nigg, BM, Hintermann, B, Goepfert, B, Dick, W, Frank, CB, . . Tscharner, V von. (2007). Muscular lower-leg asymmetry in middle-aged people. Foot and Ankle International, 28(2), 242-249. doi: 10.3113/FAI.2007.0242

van Emmerik, REA, Rosenstein, MT, McDermott, WJ, \& Hamill, J. (2004). A nonlinear dynamics approach to human movement. Journal of Applied Biomechanics, 20, 396-420. 
Winter, D. A. (2005). Kinematics, Chapter 2 Biomechanics and Motor Control of Human Movement (3rd ed.). New York: John Wiley and Sons Inc.

Young, W. (1995). Laboratory strength assessment of athletes. New Studies in Athletics, 10, 88-96.

Zifchock, RA, Davis, I, \& Hamill, J. (2006). Kinetic asymmetry in female runners with and without retrospective tibial stress fractures. Journal of Biomechanics, 39, 2792-2797. doi: 10.1016/j.jbiomech.2005.10.003 
Table 1. Differences in plantarflexor function with limb preference

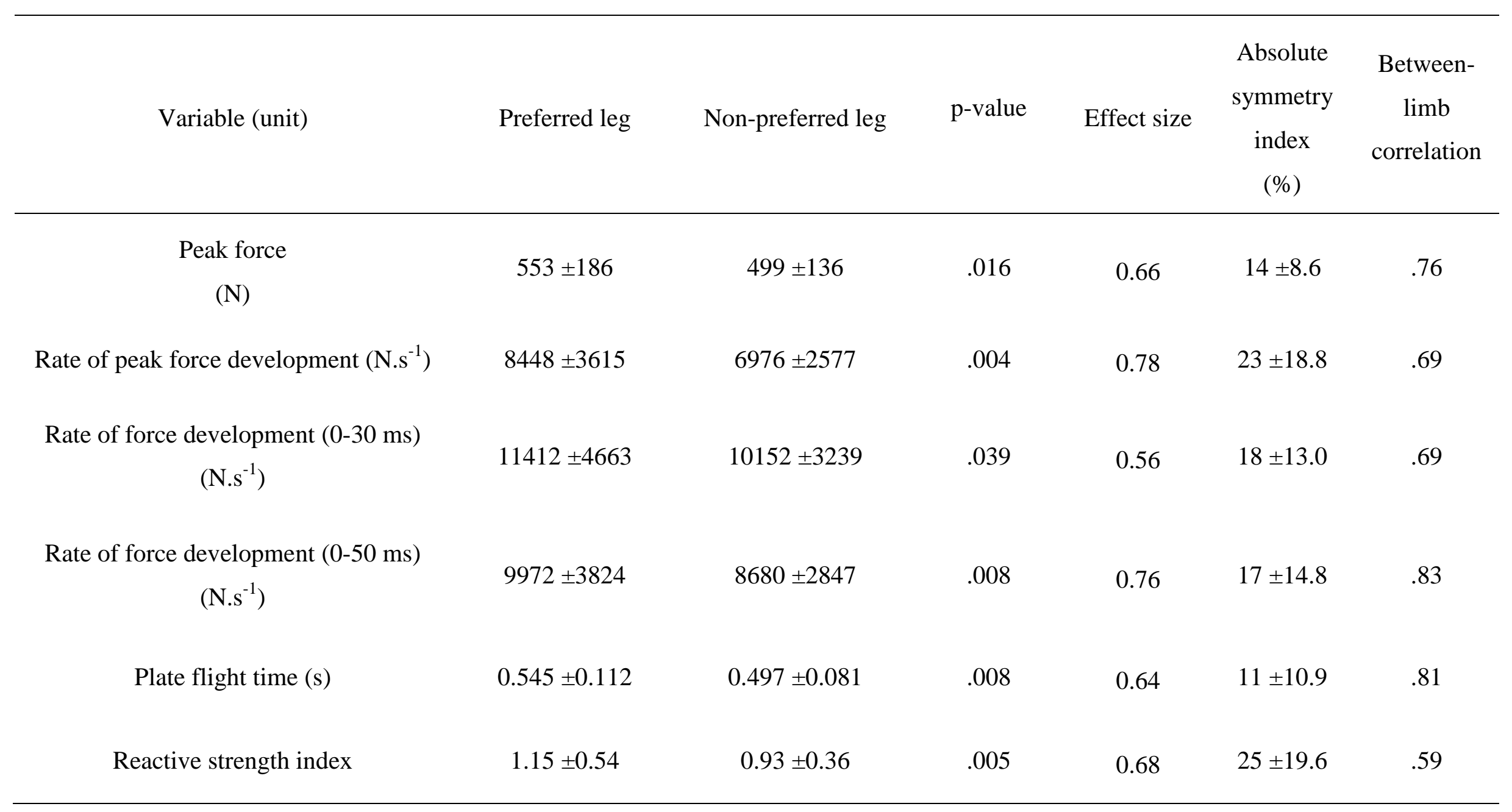




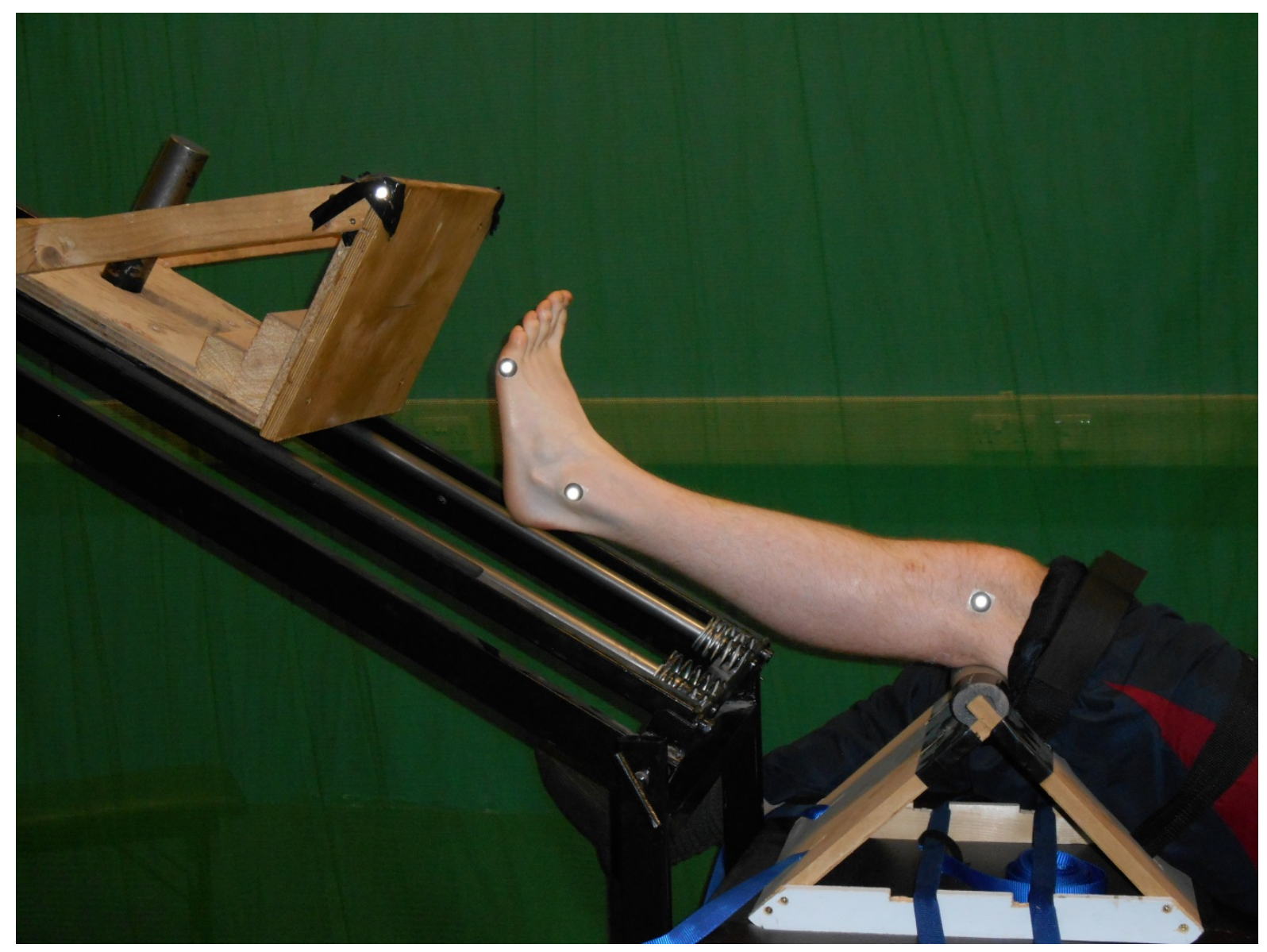

Fig. 1 Participant test position, with thigh secured and retro-reflective markers in place 